\title{
The non-equilibrium part of the inertial range in decaying homo- geneous turbulence
}

\author{
M. OBligado ${ }^{1}$ and J.C. VAssilicos ${ }^{2}$ \\ 1 Université Grenoble Alpes, CNRS, Grenoble-INP, LEGI, F-38000, Grenoble, France \\ 2 Univ. Lille, CNRS, ONERA, Arts et Métiers ParisTech, Centrale Lille, FRE 2017 - LMFL - Laboratoire de \\ Mécanique des fluides de Lille - Kampé de Feriet, F-59000 Lille, France
}

PACS 47.27.Ak - Turbulent flows, fundamentals

PACS 47.27.Gs - Isotropic turbulence; homogeneous turbulence

PACS 47.27.Jv - High-Reynolds-number turbulence

\begin{abstract}
We use two related non-stationarity functions as measures of the degree of scaleby-scale non-equilibrium in homogeneous isotropic turbulence. The values of these functions indicate significant non-equilibrium at the upper end of the inertial range. Wind tunnel data confirm Lundgren's $(2002,2003)$ prediction that the two-point separation $r$ where the second and third order structure functions are closest to their Kolmogorov scalings is proportional to the Taylor length scale $\lambda$, and that both structure functions increasingly distance themselves from their Kolmogorov equilibrium form as $r$ increases away from $\lambda$ throughout the inertial range. With the upper end of the inertial range in non-equilibrium irrespective of Reynolds number, it is not possible to justify the Taylor-Kolmogorov turbulence dissipation scaling on the basis of Kolmogorov equilibrium.
\end{abstract}

Introduction. - The Kolmogorov phenomenology relies on the presence of a statistically stationary, i.e. equilibrium, cascade of energy where large-scale energy input rate balances turbulence dissipation rate effectively instantaneously. Assuming homogeneous or locally homogeneous turbulence, and assuming external power input to be either absent or limited to wavenumbers much smaller than $k$, the interscale energy balance can be expressed as (see $9,10,20]$ ),

$$
\frac{\partial}{\partial t} K^{>}(k, t)=\Pi(k, t)-\varepsilon^{>}(k, t)
$$

where $K^{>}(k, t)=\int_{k}^{\infty} E\left(k^{\prime}, t\right) d k^{\prime}$ is the high-pass filtered kinetic energy (with $E(k, t)$ the energy spectrum), $\Pi(k, t)$ is the interscale energy flux and $\varepsilon^{>}(k, t)$ is the high-pass filtered turbulence dissipation rate.

The Kolmogorov equilibrium hypothesis is $\left|\frac{\partial}{\partial t} K^{>}(k, t)\right| \ll \varepsilon^{>}(k, t) \quad($ see $[9,10,22)$. The size of the largest turbulent eddies is typically accessed via the integral length-scale $L$, and if this equilibrium hypothesis can be extended to these largest eddies, i.e. to wavenumbers comparable to $2 \pi / L$, then

$$
\varepsilon \approx \Pi(2 \pi / L, t) \sim K^{3 / 2} / L
$$

where the scaling $K^{3 / 2} / L$ of $\Pi(2 \pi / L, t)$ comes from the expectation that there are no direct viscosity effects at the largest scales and that the memory of initial/inlet conditions has had time to fade away ( $K$ is the turbulent kinetic energy). The resulting scaling $\varepsilon=C_{\varepsilon} K^{3 / 2} / L$ where $C_{\varepsilon}=$ Const (sometimes refered to as zeroth law of turbulence or Taylor-Kolmogorov turbulence dissipation scaling) is perhaps the most important consequence of the equilibrium cascade phenomenology as it has a very wide range of implications in turbulence theory and modeling (see 22, 24, ).

However, a different dissipation scaling has been found over the past ten years in various turbulent flows, including forced periodic turbulence and decaying periodic turbulence 8 10], various types of grid-generated turbulence [24 and various turbulent shear flows [5, 17, 18, 24]:

$$
\varepsilon \sim U_{0} L_{0} K / L^{2}
$$

or equivalently $C_{\varepsilon} \sim R e_{I} / R e_{L} \sim \sqrt{R e_{I}} / R e_{\lambda}$ where $R_{L}=$ $\sqrt{K} L / \nu, R e_{\lambda}=\sqrt{K} \lambda / \nu$ and $\operatorname{Re}_{I}=U_{0} L_{0} / \nu, U_{0}$ and $L_{0}$ being initial/inlet velocity and length scales respectively ( $\nu$ is the fluid's kinematic viscosity and $\lambda$ the Taylor length-scale). One can expect this scaling to be a consequence of a cascade which is out of equilibrium, at least 
in the upper part of the inertial range (closer to $L$ ), and this was indeed confirmed by Goto \& Vassilicos (2016) 10 in direct numerical simulations of freely decaying periodic turbulence. These authors also found, in agreement with grid-generated turbulence results (see 24]), that the classical dissipation scaling $\varepsilon \sim K^{3 / 2} / L\left(C_{\varepsilon}=\right.$ Const $)$ appears rather suddenly after a number of turnover times and as the local Reynolds numbers $R_{L}$ and $R e_{\lambda}$ decrease. They also reported that this classical dissipation scaling is not a reflection of a Kolmogorov equilibrium cascade but in fact coexists with non-equilibrium over a wide range of scales, including those inertial scales closer to $L$, as in the model of Bos et al [3]. Bos et al [3] explained this non-equilibrium version of $C_{\varepsilon}=$ Const by relating it to its equilibrium version and Goto \& Vassilicos (2016) 10] explained it by noting that the three terms in equation (1) remain numerically comparable as they decay in time, and proportional to each other for $k L$ fixed close to $2 \pi$.

The question arises whether this non-equilibrium, in particular at the inertial scales closer to $L$, persists as the Reynolds number increases towards infinity. This question is addressed in this paper in the context of freely decaying homogeneous isotropic turbulence (HIT) by using published wind tunnel data and Lundgren's 14, 15 matched asymptotic expansions for the second and third order structure functions.

Finite Reynolds number effects. - We work with the Kármán-Howarth equation for decaying HIT in a rearranged version of the form found in 6,13 :

$$
-\varepsilon F=-\frac{\left\langle(\delta u)^{3}\right\rangle}{r}-\frac{4}{5} \varepsilon+\frac{6 \nu}{r} \frac{\partial}{\partial r}\left\langle(\delta u)^{2}\right\rangle
$$

where $F \equiv-\frac{3}{\varepsilon r^{5}} \int_{0}^{r} d r^{\prime} r^{\prime} \frac{\partial}{\partial t}\left\langle(\delta u)^{2}\right\rangle$ is the nonstationarity/non-equilibrium term. $\left\langle(\delta u)^{2}\right\rangle$ and $\left\langle(\delta u)^{3}\right\rangle$ are, respectively, the second and third order longitudinal structure functions defined in terms of $\delta u=u(x+r, t)-$ $u(x, t)$ where $u$ is the fluctuating velocity component in the direction of the $x$ axis, and $r$ is the distance between points $x$ and $x+r$ on that axis. This equation is the physical space equivalent of (1) where $-\varepsilon F$ and $-\frac{\left\langle(\delta u)^{3}\right\rangle}{r}$ correspond to $\frac{\partial}{\partial t} K^{>}(k, t)$ and $\Pi(k, t)$ respectively.

Finite Reynolds number (FRN) effects come from the non-stationarity/non-equilibrium term $-\varepsilon F$ and from the viscous term $\frac{6 \nu}{r} \frac{\partial}{\partial r}\left\langle(\delta u)^{2}\right\rangle 6$. It has been proved rigorously [12,23 using simple kinematic constraints on the basis that $K$ is finite that $\frac{6 \nu}{r} \frac{\partial}{\partial r}\left\langle(\delta u)^{2}\right\rangle \ll \frac{4}{5} \varepsilon$ if $r \gg \lambda$. Therefore, the viscous term contributes no significant FRN effects at scales $r \gg \lambda$.

We define the non-stationarity function $f$ by $\frac{\partial}{\partial t}\left\langle(\delta u)^{2}\right\rangle \equiv-\varepsilon f$. This function provides a direct comparison between $\frac{\partial}{\partial t}\left\langle(\delta u)^{2}\right\rangle$ and $\varepsilon$ across scales. It is related to the non-stationarity function $F$ by $F=\frac{3}{r^{5}} \int_{0}^{r} d r^{\prime} r^{\prime 4} f$ and $f=\frac{1}{3 r^{4}} \frac{d}{d r} r^{5} F$. Note that both $f$ and $F$ are dimensionless and non-negative.
It is easily seen that $f(r) \rightarrow 4 / 3$ and $F(r) \rightarrow 4 / 5$ in the limit $r \gg L$ because $\left\langle(\delta u)^{2}\right\rangle \rightarrow \frac{4}{3} K$ in that limit.

From a first order Taylor expansion and $\varepsilon=$ $15 \nu\left\langle\left(\frac{\partial u}{\partial x}\right)^{2}\right\rangle,\left\langle(\delta u)^{2}\right\rangle \approx r^{2} \frac{\varepsilon}{15 \nu}$ for $r \ll \lambda[21 . \quad$ Differentiating both sides with respect to time, we obtain $f \approx\left(\frac{r}{\lambda}\right)^{2} \frac{\lambda^{2}}{15 \nu}\left(-\frac{\frac{d}{d t} \varepsilon}{\varepsilon}\right)$ which implies $f \approx \operatorname{Const}\left(\frac{r}{\lambda}\right)^{2}$ for $r \ll \lambda$ if $K$ decays as a power of time; $F \approx \frac{3}{7} \operatorname{Const}\left(\frac{r}{\lambda}\right)^{2}$ follows under these same conditions.

We can expect the non-stationarity functions $f$ and $F$ to increase from 0 at $r=0$ to $4 / 3$ and $4 / 5$, respectively, at $r \gg L$. Incidentally, if $f$ is a monotonically increasing function of $r$, so is $F$. (Proof: $\frac{\partial}{\partial r} F>0$ if $r^{5} f>5 \int_{0}^{r} d r^{\prime} r^{4} f\left(r^{\prime}\right)$ which follows from $5 \int_{0}^{r} d r^{\prime} r^{4} f\left(r^{\prime}\right)<5 \int_{0}^{r} d r^{\prime} r^{\prime 4} f(r)=r^{5} f$ given that $f(r)>$ $f\left(r^{\prime}\right)$ for $r>r^{\prime}$.)

We might expect a tendency towards equilibrium at the smaller inertial scales, but what are the values of $f$ and $F$ for $r$ smaller than but comparable to $L$ ? The answer to this question can inform us about the degree of nonequilibrium at the larger inertial scales.

Experimental data. - We use published wind tunnel measurements of $\left\langle(\delta u)^{2}\right\rangle(r)$ and $\left\langle(\delta u)^{3}\right\rangle(r)$ for approximate HIT to calculate $F(r)$ from (4) and then $f(r)$ from $f=\frac{1}{3 r^{4}} \frac{d}{d r}\left(r^{5} F\right)$. The only published data sets that we could find for wind tunnel measurements of both $\left\langle(\delta u)^{2}\right\rangle$ and $\left\langle(\delta u)^{3}\right\rangle$ in approximate HIT conditions are from the grid turbulence experiments of Zhou \& Antonia 26], Malecot [16] and Bourgoin et al [4] and from the Modane wind tunnel measurements of $[7]$. Note that the data of Bourgoin et al 4 have been obtained in the same Modane wind tunnel as 7 . The particular datasets that we selected from these publications span the widest possible range of Reynolds numbers based on $R e_{\lambda}$. Table 1 summarises the turbulence parameters for each dataset.

All these data have been obtained with hot-wire anemometry (the specific details of the experimental setups for each value of $R e_{\lambda}$ are given in the corresponding references), although for the two larger values of $R e_{\lambda}$ the Kolmogorov length scale $\left(\eta=\left(\nu^{3} / \varepsilon\right)^{1 / 4}\right)$ was not fully resolved. In all cases, the conversion from time to space was done via the Taylor hypothesis. The Taylor lengthscale is defined from $\lambda^{2}=<u^{2}>/<(\partial u / \partial x)^{2}>$, and $R e_{\lambda}$ is calculated as $R e_{\lambda}=u^{\prime} \lambda / \nu$ where $u^{\prime} \equiv<u^{2}>^{1 / 2}$. The turbulence dissipation rate, assuming HIT, can be obtained from $\varepsilon=15 \nu<u^{2}>/ \lambda^{2}$. For the two larger values of $R e_{\lambda}$, where $\eta$ was not resolved, $\varepsilon$ and $\lambda$ have been obtained via the compensated second order structure function $\frac{\left\langle(\delta u)^{2}\right\rangle}{(\varepsilon r)^{2 / 3}}\left(R e_{\lambda}=380\right)$ or by matching the shape of $\frac{\left\langle(\delta u)^{3}\right\rangle}{(\varepsilon r)}$ at $r$ close to $\eta$ to other data $\left(R e_{\lambda}=2260\right)$. Finally, the lengthscale $L$ is calculated from the autocorrelation $R_{u u}$ as $L=\int_{0}^{\infty} R_{u u} d r$.

Results. - In figure 1 we plot the non-stationarity functions $F$ and $f$ as functions of $r / L$ and $r /\left(\lambda R e_{\lambda}\right)$. Note the relation $15 L=C_{\varepsilon} \lambda R e_{\lambda}$ in HIT, hence $\lambda R e_{\lambda}$ represents 


\begin{tabular}{|c|c|c|c|c|c|c|c|} 
Grid & $R e_{\lambda}$ & $U_{\infty}(\mathrm{m} / \mathrm{s})$ & $L(\mathrm{~cm})$ & $\eta(\mathrm{mm})$ & $\varepsilon\left(\mathrm{m}^{2} \mathrm{~s}^{-3}\right)$ & $\lambda(\mathrm{cm})$ & $u^{\prime}(\mathrm{m} / \mathrm{s})$ \\
\hline \hline ZA1 & 50 & 4.8 & 5.52 & 0.58 & 0.032 & 0.81 & 0.096 \\
ZA2 & 75 & 11.3 & 4.21 & 0.30 & 0.40 & 0.54 & 0.22 \\
ZA3 & 89 & 6.7 & 3.81 & 0.24 & 1.37 & 0.41 & 0.325 \\
Ma & 144 & 16.9 & 4 & 0.15 & 7.7 & 0.35 & 0.64 \\
MI & 380 & 34.2 & 22.0 & 0.23 & 1.19 & 0.86 & 0.63 \\
Mo & 2260 & 20.75 & $\approx 410$ & 0.30 & 0.95 & 2.80 & 1.58
\end{tabular}

Table 1: Experimental parameters : Reynolds number based on Taylor micro-scale $\left(R e_{\lambda}\right)$, incoming wind velocity $\left(U_{\infty}\right)$, integral length scale $(L)$, Kolmogorov scale $(\eta)$, energy dissipation rate $(\varepsilon)$, Taylor micro-scale $(\lambda)$ and standard deviation of fluctuating streamwise velocity $\left(u^{\prime}\right)$. ZA stands for Zhou \& Antonia 26], Ma for Malecot [16], MI for the Modane inflatable grid-turbulence experiment [4] and $M o$ for the Modane measurements of Gagne et al 7 ]

the integral scale too but without FRN effects coming from $C_{\varepsilon} . F$ is found to be larger than 0.2 and $f$ larger than 0.4 for all $r$ larger or equal to $L / 5$ in all data sets. In the case of our highest Reynolds number, $R e_{\lambda}=2260, r=40 \lambda$ corresponds to $r \approx L / 4$, which shows that $L / 5$ is quite a small scale for all our data and well within the smallest scales of the inertial range for most if not all of them. We must conclude that most if not all the inertial range is not in Kolmogorov equilibrium in all our data sets, and definitely not in the part of the inertial range closer to its upper bound $L$. Justifying $C_{\varepsilon}=$ Const on the basis of such equilibrium at the Reynolds numbers considered here is definitely questionable.

Note the much better collapse with $\lambda R e_{\lambda}$ than with $L$ for both $F$ and $f$ in figure 1 . This might suggest that the lack of collapse with $L$ is caused by the residual Reynolds number dependence of $C_{\varepsilon}$ at the smaller $R e_{\lambda}$ values. Extrapolating to $R e_{\lambda}$ larger than 2260 might suggest that $F$ and $f$ remain larger than 0.2 and 0.4 , respectively, for $r \geq L / 5$ as $R e_{\lambda}$ grows beyond 2260. A non-stationarity function $f$ larger than 0.4 means that $-\frac{\partial}{\partial t}\left\langle(\delta u)^{2}\right\rangle>0.4 \varepsilon$. Hence, the current state of experimental evidence for HIT does not rule out, and in fact might even suggest, that the upper part of the inertial range between $L$ and an order of magnitude smaller than $L$ is out of equilibrium at all Reynolds numbers. This conclusion is supported by Lundgren's 14 matched asymptotic expansions for the second and third order structure functions which we examine next.

Extrapolation to even higher $R e_{\lambda}$. Lundgren 14,15 obtained the following forms for $\left\langle(\delta u)^{2}\right\rangle$ and $\left\langle(\delta u)^{3}\right\rangle$ from the Navier-Stokes equation, and in particular the KármánHowarth equation, by using the method of matched asymptotic expansions in the range $\eta \ll r \ll L$ :

$$
\begin{aligned}
& \frac{\left\langle(\delta u)^{2}\right\rangle}{(\varepsilon r)^{2 / 3}} \approx C\left[1-A_{2}(r / L)^{2 / 3}-B_{2}(r / \eta)^{-4 / 3}\right] \\
- & \frac{\left\langle(\delta u)^{3}\right\rangle}{(\varepsilon r)} \approx 4 / 5-A_{3}(r / L)^{2 / 3}-B_{3}(r / \eta)^{-4 / 3}
\end{aligned}
$$

where $A_{2}, B_{2}, A_{3}$ and $B_{3}$ are dimensionless constants of order 1 , and $C=2$ from widely accepted experimental
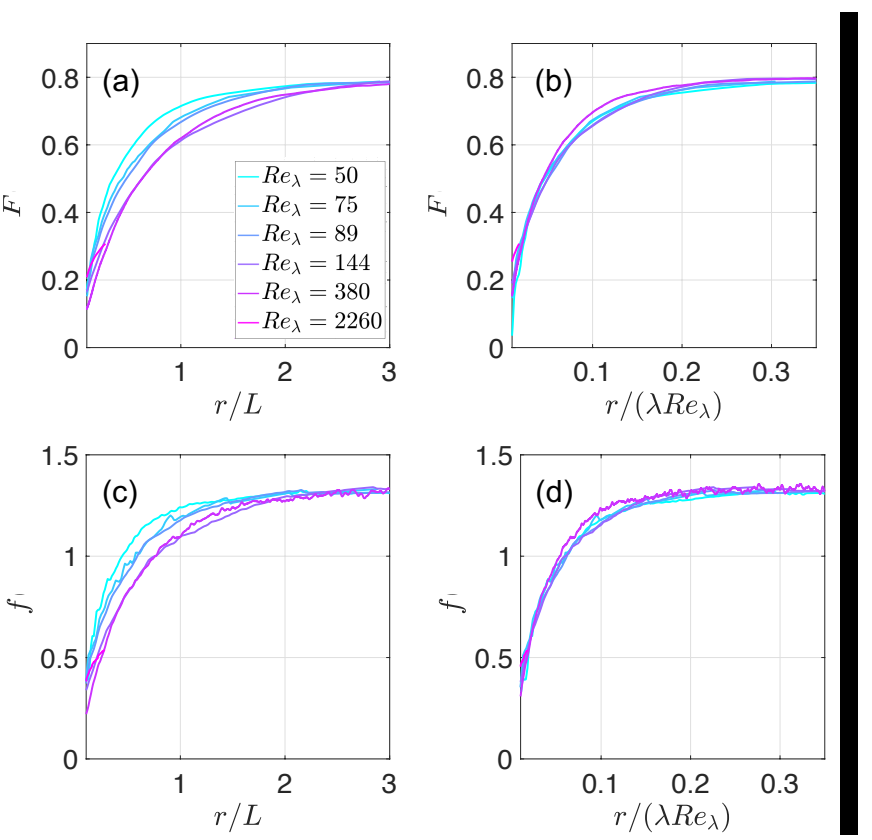

Fig. 1: Top plots: non-stationarity function $F$ vs (a) $r / L$ and (b) $r /\left(\lambda R e_{\lambda}\right)$. Bottom plots: non-stationarity function $f$ vs (c) $r / L$ and (d) $r /\left(\lambda R e_{\lambda}\right)$. The colour code for $R e_{\lambda}$ indicated in (a) is the same in all the plots in this paper and the data sets used to obtain $F$ and $f$ are described in Table 1 .

evidence to date (e.g. see Pope $2000[19$ ). It is worth mentioning that these corrections to the Kolmogorov equilibrium scalings of $\left\langle(\delta u)^{2}\right\rangle$ are different from the nonequilibrium correction obtained by Yoshizawa 25] from his spectral closure theory and by Bos \& Rubinstein [2] who obtained the same correction as Yoshizawa by using Kovaznays spectral closure model. Whilst the correction of these authors scales as $r^{2 / 3}$ similarly to the correction $A_{2}(r / L)^{2 / 3}$ in (5), it also scales with $d \varepsilon / d t$ which is not generally the case in (5) (unless we assume, for example, that the $K-\varepsilon$ equation for $d \varepsilon / d t$ and $C_{\varepsilon}=$ Const hold true). More importantly, however, the non-equilibrium correction of Yoshizawa and Bos \& Rubinstein does not capture the $B_{2}(r / \eta)^{-4 / 3}$ correction in (5) which is essen- 

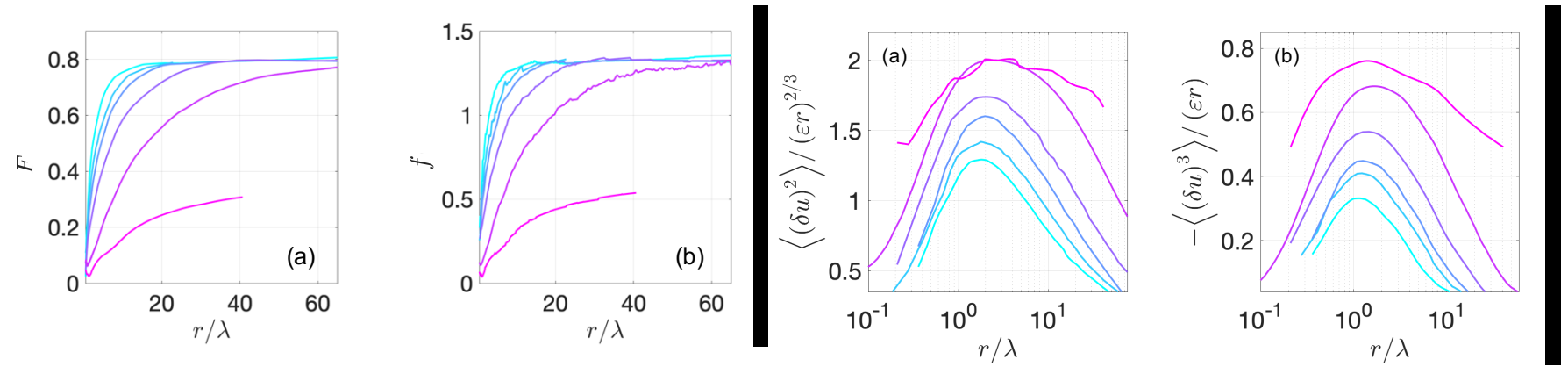

Fig. 2: Functions (a) $F$ and (b) $f$, both plotted vs $r / \lambda$. The colour code for different $R e_{\lambda}$ is the same as in figure 1 and the data sets used to obtain $F$ and $f$ are described in Table 1.

tial for our conclusions as the special role of the Taylor microscale described below cannot be captured without it.

Using (1), formulae (4) and (5) lead to

$$
\begin{aligned}
F \approx & {\left[A_{3}(r / L)^{2 / 3}-4 C R e_{\lambda}^{-2}(r / L)^{-4 / 3}+\right.} \\
& \left.8 C A_{2} R e_{\lambda}^{-2}(r / L)^{-2 / 3}\right]- \\
& B_{3} R e_{\lambda}^{-2 / 3}(r / \lambda)^{-4 / 3}\left[1+4 C \frac{B_{2}}{B_{3}} \operatorname{Re}_{\lambda}^{-2}(r / L)^{-4 / 3}\right] .
\end{aligned}
$$

This expression for $F$ implies that $F \approx A_{3}(r / L)^{2 / 3}$ in the limit $R e_{\lambda} \rightarrow \infty$ as $r / L$ is kept constant or as $r$ and $t$ are both kept constant relative to laboratory length and time scales. In other words, the higher part of the inertial range does not tend towards equilibrium as $R e_{\lambda} \rightarrow \infty$, it keeps its level of non-equilibrium unchanged.

This observation does not contradict the fact that, as $R e_{\lambda} \rightarrow \infty$ and $L / \lambda \rightarrow \infty$, an approximate equilibrium range does develop in the limit where $r / L \rightarrow 0$. Indeed, for fixed $r / \lambda, F$ does tend to 0 as $R e_{\lambda} \rightarrow \infty$, and our data show evidence in agreement with this in figure 2, note how both $F$ and $f$ decrease, presumably towards zero, as $R e_{\lambda}$ increases while keeping $r / \lambda$ constant. However, this asymptotic equilibrium part of the inertial range is so far from $L$, and increasingly so with increasing $R e_{\lambda}$, that it cannot be used as a basis for $C_{\varepsilon}=$ Const.

To clarify this distinction between the asymptotic equilibrium scales at the lower end of the inertial range and the non-equilibrium scales at the upper end of the inertial range we calculate the value $r_{\max }$ of $r$ where Lundgren's formulae for $\frac{\left\langle(\delta u)^{2}>\right.}{(\varepsilon r)^{2 / 3}}$ and $-\frac{\left\langle(\delta u)^{3}\right\rangle}{(\varepsilon r)}$ have a maximum. As already noted in 15], the maximum is at $r_{\max } \sim \lambda$ for both $\frac{\left\langle(\delta u)^{2}\right\rangle}{(\varepsilon r)^{2 / 3}}$ and $-\frac{\left\langle(\delta u)^{3}\right\rangle}{(\varepsilon r)}$. Our data confirm this prediction and give $r_{\max } \approx 2 \lambda$ for $\frac{\left\langle(\delta u)^{2}\right\rangle}{(\varepsilon r)^{2 / 3}}$ and $r_{\max } \approx 1.5 \lambda$ for $-\frac{\left\langle(\delta u)^{3}\right\rangle}{(\varepsilon r)}$, as shown in figures $3 a \& b$. To our knowledge, this is the first time that this prediction is confirmed for the second order structure function and the first time that it is confirmed over such a wide range of Reynolds num-

Fig. 3: Compensated (a) second-order structure function $\frac{\left\langle(\delta u)^{2}\right\rangle}{(\varepsilon r)^{2 / 3}}$ and (b) third-order structure function $\frac{\left\langle(\delta u)^{3}\right\rangle}{\varepsilon r}$, both plotted vs $r / \lambda$.

bers for $-\frac{\left\langle(\delta u)^{3}\right\rangle}{(\varepsilon r)}$ (data from only two Reynolds numbers from Yves Gagne's Modane measurements were used in 15 ).

It may be worth pointing out that the empirical formula for $\left\langle(\delta u)^{2}\right\rangle$ in 1, 11], namely

$$
\frac{\left\langle(\delta u)^{2}\right\rangle}{(\varepsilon \eta)^{2 / 3}}=\frac{(r / \eta)^{2}(1+r / L)^{-2 / 3}}{15\left[1+D(r / \eta)^{2}\right]^{2 / 3}}
$$

where $30 D^{2 / 3}=1$, leads to a maximum of $\frac{\left\langle(\delta u)^{2}\right\rangle}{(\varepsilon r)^{2 / 3}}$ at $r_{\max } \approx 2^{5 / 6} C_{\varepsilon} \lambda$ for $L / \eta \gg 1$ (using $\varepsilon=C_{\varepsilon} u^{\prime 3} / L=$ $15 \nu u^{\prime 2} / \lambda^{2}$ to relate $\lambda$ to $L$ via $\left.15 L / \lambda=C_{\varepsilon} R e_{\lambda}\right)$. This empirical formula is designed by construction to give $<(\delta u)^{2}>\approx 2(\varepsilon r)^{2 / 3}$ in the range $\eta \ll r \ll L$, and the right kinematic behaviours at $r / L \gg 1$ and $r / \eta \ll 1$. It is interesting that it combines dependencies on $L$ and $\eta$ in exactly the right way for $\frac{\partial}{\partial r} \frac{\left\langle(\delta u)^{2}\right\rangle}{(\varepsilon r)^{2 / 3}}=0$ to yield $r_{\text {max }} \sim \lambda$, in agreement with Lundgren's prediction.

Conclusions. - Given that the inertial range is, by definition, a range where the viscosity is effectively absent, it must be defined as $\lambda \ll r \ll L$. Our data and Lundgren's 14,15] formulae (5) and (6) show that $\left\langle(\delta u)^{2}\right\rangle$ and $\left\langle(\delta u)^{3}\right\rangle$ are closest to their Kolmogorov equilibrium predictions at the lower end of the inertial range, i.e. $r=r_{\max } \sim \lambda$. Both $\left\langle(\delta u)^{2}\right\rangle$ and $\left\langle(\delta u)^{3}\right\rangle$ increasingly deviate away from their Kolmogorov equilibrium prediction as $r$ increases in the inertial range. The matched asymptotic expansions (5) and (6) imply that $\left\langle(\delta u)^{2}\right\rangle$ and $\left\langle(\delta u)^{3}\right\rangle$ tend towards $C(\varepsilon r)^{2 / 3}$ and $-\frac{4}{5} \varepsilon r$, respectively, as $R e_{\lambda} \rightarrow \infty$ for fixed $r / \lambda$ but not for fixed $r / L$. For fixed $r / L$ smaller that 1 , they tend to

$$
\left\langle(\delta u)^{2}\right\rangle=C(\varepsilon r)^{2 / 3}\left[1-A_{2}(r / L)^{2 / 3}\right]
$$

and

$$
\left\langle(\delta u)^{3}\right\rangle=-(\varepsilon r)\left[4 / 5-A_{3}(r / L)^{2 / 3}\right] .
$$

The upper end of the range is therefore always significantly out of equilibrium whatever the Reynolds number. 
The wind tunnel data that we have compiled here for HIT support this conclusion and suggest that the upper end of the inertial range where $r$ is up to one order of magnitude smaller than $L$ is such that $-\frac{\partial}{\partial t}\left\langle(\delta u)^{2}\right\rangle$ is comparable to $\varepsilon$. Any justification of $C_{\varepsilon}=$ Const in terms of Kolmogorov equilibrium is therefore, at the very least, seriously questionable in decaying HIT, at any Reynolds number.

$$
* * *
$$

This work was motivated by a discussion with Claude Cambon during a dinner held in his honour in Lyon to mark his 65 th year.

\section{REFERENCES}

[1] RA Antonia and Paolo Burattini. Approach to the 4/5 law in homogeneous isotropic turbulence. Journal of fluid mechanics, 550:175-184, 2006.

[2] W J T Bos and R Rubinstein. Dissipation in unsteady turbulence. Physical Review Fluids, 2(2):022601(R), 2017.

[3] WJT Bos, L Shao, and JP Bertoglio. Spectral imbalance and normalized dissipation rate of turbulence. Physics of fluids, 19(4):045101, 2007.

[4] M Bourgoin, C Baudet, S Kharche, N Mordant, T Vandenberghe, S Sumbekova, N Stelzenmuller, A Aliseda, $\mathrm{M}$ Gibert, P-E Roche, et al. Investigation of the smallscale statistics of turbulence in the modane s1ma wind tunnel. CEAS Aeronautical Journal, 9(2):269-281, 2018.

[5] G Cafiero and JC Vassilicos. Non-equilibrium turbulence scalings and self-similarity in turbulent planar jets. Proceedings of the Royal Society A, 475(2225):20190038, 2019.

[6] L Danaila, F Anselmet, Tongming Zhou, and RA Antonia. A generalization of yaglom's equation which accounts for the large-scale forcing in heated decaying turbulence. Journal of Fluid Mechanics, 391:359-372, 1999.

[7] Yves Gagne, Bernard Castaing, Christophe Baudet, and Yann Malécot. Reynolds dependence of third-order velocity structure functions. Physics of Fluids, 16(2):482-485, 2004.

[8] Susumu Goto and J Christos Vassilicos. Energy dissipation and flux laws for unsteady turbulence. Physics Letters A, 379(16-17):1144-1148, 2015.

[9] Susumu Goto and JC Vassilicos. Local equilibrium hypothesis and taylor's dissipation law. Fluid Dynamics Research, 48(2):021402, 2016.

[10] Susumu Goto and JC Vassilicos. Unsteady turbulence cascades. Physical Review E, 94(5):053108, 2016.

[11] Susan Kurien and Katepalli R Sreenivasan. Anisotropic scaling contributions to high-order structure functions in high-reynolds-number turbulence. Physical Review E, 62(2):2206, 2000.

[12] Sylvain Laizet, JC Vassilicos, and Claude Cambon. Interscale energy transfer in decaying turbulence and vorticitystrain-rate dynamics in grid-generated turbulence. Fluid Dynamics Research, 45(6):061408, 2013.

[13] LD Landau and EM Lifschitz. Fluid mechanics, series in advanced physics, vol. 6, 1959.
[14] Thomas S Lundgren. Kolmogorov two-thirds law by matched asymptotic expansion. Physics of fluids, 14(2):638-642, 2002.

[15] Thomas S Lundgren. Kolmogorov turbulence by matched asymptotic expansions. Physics of fluids, 15(4):10741081, 2003.

[16] Yann Malécot. Intermittence en turbulence 3D: statistique de la vitesse et de la vorticité. $\mathrm{PhD}$ thesis, Université Joseph Fourier (Grenoble), 1998.

[17] Jovan Nedić, Stavros Tavoularis, and Ivan Marusic. Dissipation scaling in constant-pressure turbulent boundary layers. Physical Review Fluids, 2(3):032601, 2017.

[18] Martin Obligado, T Dairay, and John Christos Vassilicos. Nonequilibrium scalings of turbulent wakes. Physical Review Fluids, 1(4):044409, 2016.

[19] Stephen B Pope. Turbulent flows. Turbulent Flows, by Stephen B. Pope, pp. 806. ISBN 0521591252. Cambridge, UK: Cambridge University Press, September 2000., page 806, 2000.

[20] Pierre Sagaut and Claude Cambon. Homogeneous turbulence dynamics, volume 897. Springer, 2nd edition edition, 2018.

[21] Geoffrey Ingram Taylor. Statistical theory of turbulence. iv. diffusion in a turbulent air stream. Proceedings of the Royal Society of London. Series A-Mathematical and Physical Sciences, 151(873):465-478, 1935.

[22] Hendrik Tennekes and John Leask Lumley. A first course in turbulence. MIT press, 1972.

[23] PC Valente and JC Vassilicos. The energy cascade in gridgenerated non-equilibrium decaying turbulence. Physics of Fluids, 27(4):045103, 2015.

[24] J Christos Vassilicos. Dissipation in turbulent flows. Annual Review of Fluid Mechanics, 47:95-114, 2015.

[25] A Yoshizawa. Nonequilibrium effect of the turbulentenergy-production process on the inertial-range energy spectrum. Physical Review E, 49(5):4065, 1994.

[26] Tongming Zhou and RA Antonia. Reynolds number dependence of the small-scale structure of grid turbulence. Journal of Fluid Mechanics, 406:81-107, 2000. 